\title{
Accurate Atom Counting in Mesoscopic Ensembles
}

\author{
D. B. Hume, I. Stroescu, M. Joos, W. Muessel, H. Strobel, and M. K. Oberthaler* \\ Kirchhoff-Institute for Physics, University of Heidelberg, INF 227, 69120 Heidelberg, Germany
}

(Received 29 July 2013; published 16 December 2013)

\begin{abstract}
Many cold atom experiments rely on precise atom number detection, especially in the context of quantum-enhanced metrology where effects at the single particle level are important. Here, we investigate the limits of atom number counting via resonant fluorescence detection for mesoscopic samples of trapped atoms. We characterize the precision of these fluorescence measurements beginning from the single-atom level up to more than one thousand. By investigating the primary noise sources, we obtain single-atom resolution for atom numbers as high as 1200. This capability is an essential prerequisite for future experiments with highly entangled states of mesoscopic atomic ensembles.
\end{abstract}

DOI: 10.1103/PhysRevLett.111.253001

The Heisenberg uncertainty principle sets a fundamental limit $\Delta \phi=1 / N$ on the precision at which one can determine an interferometric phase $\phi$ using $N$ particles [1]. A prerequisite for reaching the Heisenberg-limited uncertainty in a real measurement is a particle detector with atom number variance $\sigma_{N}^{2} \ll 1$, i.e., exact particle counting at the interferometer output. This capability is challenging to realize, particularly for large particle numbers. For example, single-photon detectors suffer from limited quantum efficiency (typically $<95 \%$ ), which prohibits resolving photon numbers for $N \gg 10$ [2]. On the other hand, single atoms can be detected with near-unit efficiency by trapping them and observing their fluorescence [3]. Here, we extend this single-atom counting capability to mesoscopic atom numbers by high accuracy fluorescence measurements.

One example where single-atom resolution becomes necessary is spectroscopy with maximally entangled states. Here, it has been shown that Heisenberg-limited precision requires measurement of the parity [4]. Another example is interferometry with spin-squeezed atomic states [5], where experimental results have shown a reduction of atom number variance approaching a level at which singleatom resolution becomes relevant [6]. Similarly, such high resolution atom detection would allow the direct observation of twin atom pairs produced via spin-changing collisions [7-9] and enable their use for interferometry at the Heisenberg limit.

The most common detection method for neutral atoms is absorption imaging, but the precision of such measurements on mesoscopic ensembles has thus far been limited to the level of a few atoms $[10,11]$. Single-atom resolution for small atom numbers $(N \sim 10)$ has been achieved by fluorescence detection of neutral atoms in free space [12] as well as in magneto-optical traps (MOTs) [13-15], optical dipole traps [16], and optical cavities [17-19]. A recent experiment explored the detection of mesoscopic ensembles of atoms [20] in an optical cavity, and stability at the single-atom level was observed in repeated measurements
PACS numbers: 37.10.De, 32.50.+d, 37.10.Gh, 42.50.Lc

for effective atom numbers as high as $N=150$. In this case, however, accurate determination of the absolute atom number was not possible due to inhomogeneous coupling to the standing-wave probe light. On the other hand, spatially resolved fluorescence measurements of atoms in optical lattices can determine the number of singly occupied sites [21-23], but atom pairs are quickly lost due to light-assisted collisions. In contrast, here, we show exact counting of the total atom number in mesoscopic ensembles by fluorescence measurements in a MOT (see Fig. 1). (a)
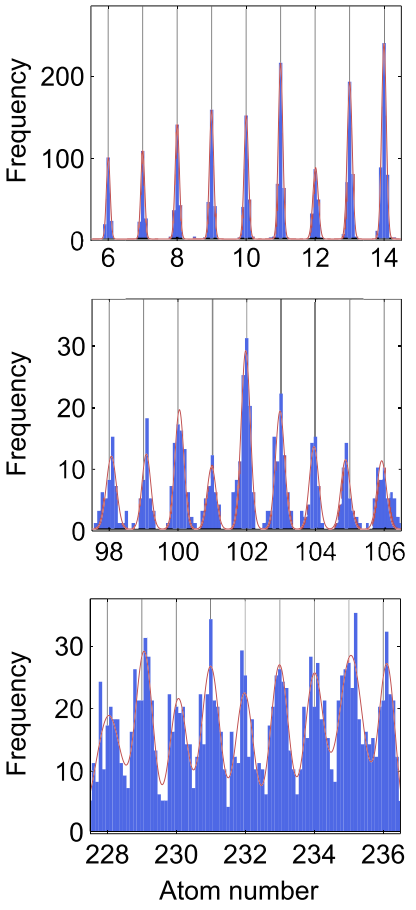

(b)
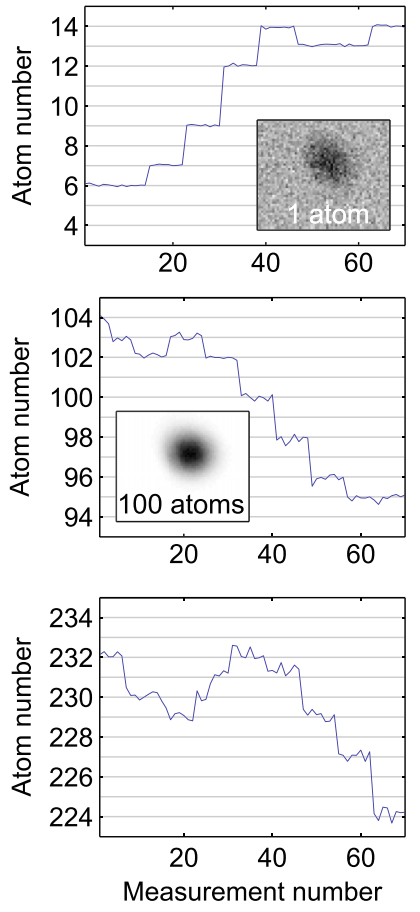

FIG. 1 (color online). (a) Histogram of collected fluorescence signal (detection time $t=100 \mathrm{~ms}$ ) and Gaussian fits to the resulting distributions (red lines). (b) Example time traces for different fluorescence levels. The upper and center insets show the signal of a single atom and 100 atoms, respectively. 
To estimate the limits of this approach, consider a fluorescence measurement of $N$ trapped atoms. Two competing noise sources, fluorescence noise and noise from atom loss, determine the maximum atom number $N_{\max }$ for which single-atom resolution is possible. Photon shot noise (PSN) contributes a variance of $N / n_{p}$ in terms of atom number when detecting $n_{p}$ photons per atom in the absence of background photons. The signal per atom can be expressed by $n_{p}=R_{\mathrm{sc}} \eta t$, where $R_{\mathrm{sc}}$ is the photon scattering rate, $\eta$ is the overall photon detection efficiency, and $t$ is the detection time. The second noise source, atom number fluctuations due to trap loss, contributes a variance of $N t / 2 \tau$, where $\tau$ is the trap lifetime. We can determine the optimal $t$ by minimizing the total variance $\sigma_{N}^{2}=N / \eta R_{\mathrm{sc}} t+N t / 2 \tau$. Here we find $t_{\mathrm{opt}}=\sqrt{2 \tau / \eta R_{\mathrm{sc}}}$, which is independent of the atom number. Furthermore, by setting $\sigma_{N}=1$ we calculate an upper bound $N_{\max }=\sqrt{\tau \eta R_{\mathrm{sc}} / 2}$. As a concrete example, consider ${ }^{87} \mathrm{Rb}(\Gamma / 2 \pi=6.1 \mathrm{MHz})$ trapped in a MOT. Each atom fluoresces at a rate $R_{\mathrm{sc}}=\Gamma / 2 \times s_{0} /\left(1+s_{0}+4 \Delta^{2} / \Gamma^{2}\right)$, where $s_{0}$ is the saturation parameter and $\Delta$ is the detuning of the laser from resonance. If we assume typical experimental parameters $\left(s_{0}=1, \Delta=-\Gamma / 2, \eta=0.01\right.$, and $\tau=100 \mathrm{~s}$ ), we find $t_{\mathrm{opt}}=56 \mathrm{~ms}$ and $N_{\mathrm{max}}=1800$. This number is at least 2 orders of magnitude higher than the atom numbers counted in previous neutral-atom experiments. In what follows we show measurements approaching this limit.

In our experiment, we image a MOT of ${ }^{87} \mathrm{Rb}$ atoms onto a low-noise CCD camera. We estimate the total efficiency of the imaging system to be $\eta=0.01$, which includes the numerical aperture of the aspherical objective lens (NA = 0.23 ), the camera quantum efficiency, and the transmission of all optical elements. The MOT beam diameters typically have a waist of $w=1.5 \mathrm{~mm}$ during atom counting and the peak intensity, summed over all six beams, is $23 \mathrm{~mW} / \mathrm{cm}^{2}$, corresponding to a saturation parameter $s_{0} \simeq 6.5$. From this, we estimate the scattering rate per atom as $R_{\mathrm{sc}} \simeq 15 \times 10^{6} \mathrm{~s}^{-1}$ at the detuning of approximately $-\Gamma / 2$.

The histograms in Fig. 1 are generated by binning repeated fluorescence measurements over an 8 hour time period. The effective detection time for the measurements is $100 \mathrm{~ms}$, where each measurement integrates the fluorescence from two adjacent $50 \mathrm{~ms}$ exposures. The background count level, recalibrated every 15 minutes, is typically less than the signal from three atoms. For atom numbers as high as $N \sim 300$, resolved peaks appear in the fluorescence histograms corresponding to the signal from an exact number of atoms. Over the same range of atom numbers, steps can be observed in the time-resolved fluorescence signal, coinciding with the loading or loss of individual atoms. These features indicate atom number resolution significantly below the single-atom level.

Based on the resolved histogram peaks, we can characterize several properties of the detector. First, by fitting the peaks to a sum of equally spaced Gaussian distributions, we calibrate the single-atom count rate to be 90310 counts/atom/s. A quadratic fit to the centroid of all resolvable peaks as a function of $N$ reveals no evidence for nonlinear scaling of the count rate with atom number. The uncertainty in the second-order fit coefficient is one way to quantify the calibration accuracy, and we constrain the deviation from linearity to below $0.02 \%$ at $N=250$ (95\% confidence interval). The widths of the individual distributions are a measure of fluorescence noise for a given atom number. For example, at $N=100$ we find a standard deviation of $\sigma=0.14$ atoms growing to $\sigma=0.27$ atoms at $N=230$. These numbers can be compared to the expected photon shot noise of $\sigma_{\mathrm{PSN}}=0.11$ at $N=100$ and $\sigma_{\mathrm{PSN}}=0.16$ at $N=230$.

To characterize the detection noise $\sigma_{N}$ in a general way for higher atom numbers, we calculate the two-sample atom variance (equivalent to the Allan variance in frequency measurements)

$$
\sigma_{N}^{2}=\frac{1}{2}\left\langle\left(S_{n+1}-S_{n}\right)^{2}\right\rangle,
$$

where $S_{n}$ and $S_{n+1}$ are the signals of consecutive measurements, each integrating CCD counts for time $t$. This measurement captures both fluorescence noise and number fluctuations due to atom loss, but, in contrast to the histograms above, is not susceptible to long term drifts in the

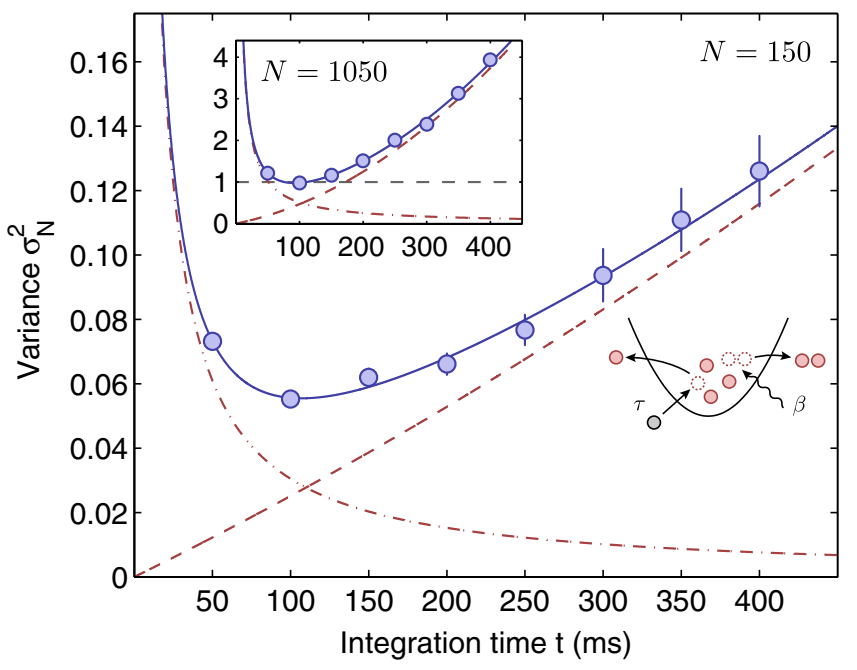

FIG. 2 (color online). Atom number variance as a function of exposure time. For atom numbers in the range $N=100$ to 200, the measured variance (circles) reaches a minimum near $t=100 \mathrm{~ms}$. For short integration times $\sigma_{N}^{2}$ is limited by photon shot noise and additional fluorescence noise, which average in time (dash-dot line). For long integration times, the finite lifetime is the dominant noise contribution (dashed line), where the main loss processes, depicted in the diagram, are collisions with background gas and light-assisted collisions. Higher atom numbers $(N=1000$ to 1100 in the inset) exhibit a higher overall variance, but the optimal detection time is found to be similar. The error bars represent 1- $\sigma$ statistical uncertainties and the solid lines are fits based on the model described in the text. 
signal. Figure 2 shows the results of such an analysis for atom numbers in the range $N=100$ to 200 . It indicates that there is an optimal detection time $t_{\mathrm{opt}} \sim 100 \mathrm{~ms}$, after which atom loss begins to dominate the noise. The same analysis for $N=1000$ to 1100 (see Fig. 2, inset) shows that $t_{\text {opt }}$ does not change significantly over the full range of atom numbers, as expected.

Taking the $100 \mathrm{~ms}$ detection time, we determine the variance as a function of atom number up to $N=1200$ (see Fig. 3). Here, it can be seen that the single-atom resolution threshold $\sigma_{N}=1$ is reached near $N=1080$. Viewing the same data on a logarithmic plot (see Fig. 3, inset) shows how the variance initially scales with $N$ at low atom numbers then changes to scaling with $N^{2}$ at higher atom numbers.

To better understand the limiting noise sources we fit an equation of the following form to the data:

$$
\sigma_{N}^{2}=a(N) t^{-1}+b(N) t+c(N) t^{2},
$$

where the first term represents fluorescence noise and Photon shot noise, while the last two terms come from atom loss-both the atom shot noise due to discrete loss events, quantified by $b(N)$, and the decay of the mean number of atoms, quantified by $c(N)$. In particular, we use $a(N)=\left(N / \eta R_{\mathrm{sc}}\right)+(\alpha N)^{2}$, where the first term represents photon shot noise and the second term is an additional noise source that is assumed to be uncorrelated in time but common to all atoms in the MOT. This describes,

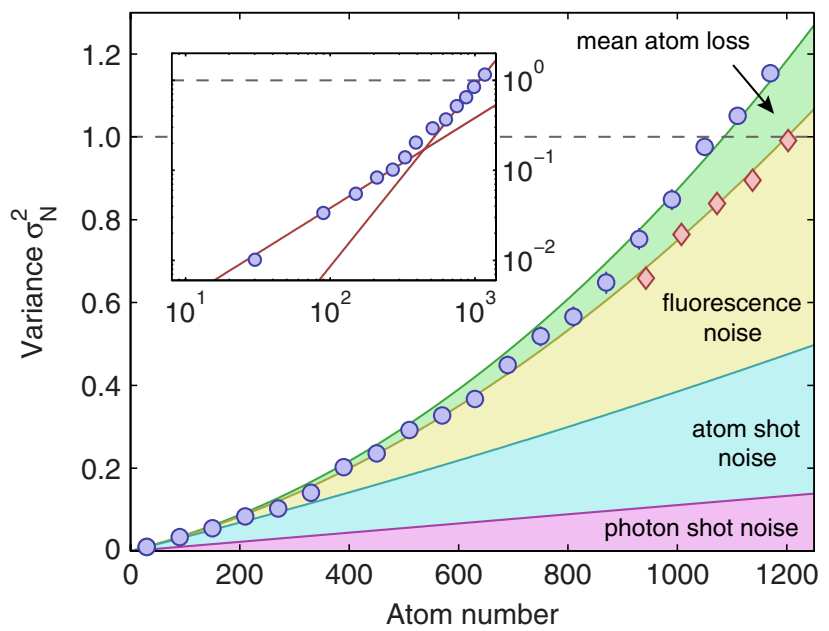

FIG. 3 (color online). Single-atom resolution for mesoscopic atom numbers. For the near-optimal exposure time of $100 \mathrm{~ms}$ the variance $\sigma_{N}^{2}$ reaches the limit of single-atom resolution at $N=1080$. A fit to the data based on a noise model incorporating fluorescence noise and atom loss (upper green line) allow us to estimate the relative contribution of these noise sources (shaded regions) and compensate for the mean atom loss (red diamonds) as described in the text. A logarithmic plot (inset) shows how the scaling of the variance with respect to atom number transitions from linear to quadratic as the additional fluorescence noise begins to dominate the shot noise. for example, fast frequency or intensity noise on the MOT laser beams. To determine $b(N)$ and $c(N)$ we use a master equation approach based on the rate equation $d N / d t=-N / \tau-\beta N^{2}$, where both one-body loss, parametrized by $\tau$, and two-body loss, parametrized by $\beta$, are considered (see the Supplemental Material [24]). The resulting noise coefficients are given by $b(N)=N / 2 \tau+\beta N^{2}$, which is the dominant effect of loss in our measurements, and $c(N)=\left(N / \tau+\beta N^{2}\right)^{2} / 2$.

A fit of the noise model to the data, for which we vary $\alpha$, $\tau$, and $\beta$, is performed individually for each atom number. Two examples of such fits are shown in Fig. 2. We take the means of the independent fit parameters as inputs to the noise model to produce the curve in Fig. 3. We extract a value for the fluorescence noise parameter of $\alpha=1.9(1) \times 10^{-4} \mathrm{~s}^{1 / 2}$ (uncertainties represent $1-\sigma$ statistical uncertainty). A likely source of this additional noise in our experiment is frequency noise on the MOT lasers, which would correspond to about $10 \mathrm{kHz}$ deviations in detuning when averaged over the detection time. We also extract the loss parameters $\tau=246(44) \mathrm{s}$ and $\beta=3(3) \times 10^{-7} \mathrm{~s}^{-1}$, indicating that light-assisted collisions contribute only a small amount to the detection errors at these atom numbers. Since the atom loss is well known for all atom numbers based on the calibrated parameters, we can improve the measurement accuracy by compensating for the loss that occurs during detection. If the raw measurement yields the result $N$, then one computes a losscompensated result $N^{\prime}=N+N t / 2 \tau$, neglecting $\beta$. Assuming proper measurement calibration, the limiting noise is then given by $\operatorname{Var}\left(S_{n+1}-S_{n}\right) / 2$. Computing this variance for the same data set yields a threshold for single atom resolution of $N=1200$, coinciding with the sum of remaining noise terms, as shown in Fig. 3. To illustrate the meaning of these variances in terms of atom counting, consider the fidelity, here defined as the probability of exactly identifying the initial atom number. We evaluate this based on a Monte Carlo simulation assuming the measured count rate, fluorescence noise, and loss parameters, and find a fidelity of $99.8 \%$ at $N=10,98.5 \%$ at $N=100$, and $44 \%$ at $N=1200$ (for details see the Supplemental Material [24]).

We now investigate state-selective detection of the two hyperfine levels, $|F=1\rangle$ and $|F=2\rangle$, in the ${ }^{2} S_{1 / 2}$ manifold (see Fig. 4). The technique is based on release and recapture of the atoms, where, during the release, atoms in $|F=2\rangle$ are pushed out of the capture volume by resonant radiation pressure. As a starting point, we measure the efficiency of the release and recapture, as a function of the release time, without radiation pressure. By counting the atoms before and after the release, we find a recapture fidelity above $99.92 \%$ for release times up to $2 \mathrm{~ms}$. To distinguish the populations we apply a laser pulse resonant with the $|F=2\rangle \rightarrow\left|{ }^{2} P_{3 / 2}, F=3\right\rangle$ transition during the release time (between two exposures in a single CCD 
(a)

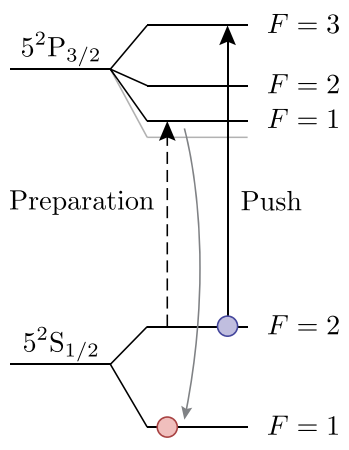

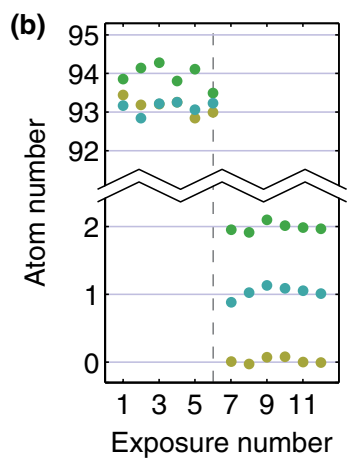

(c)

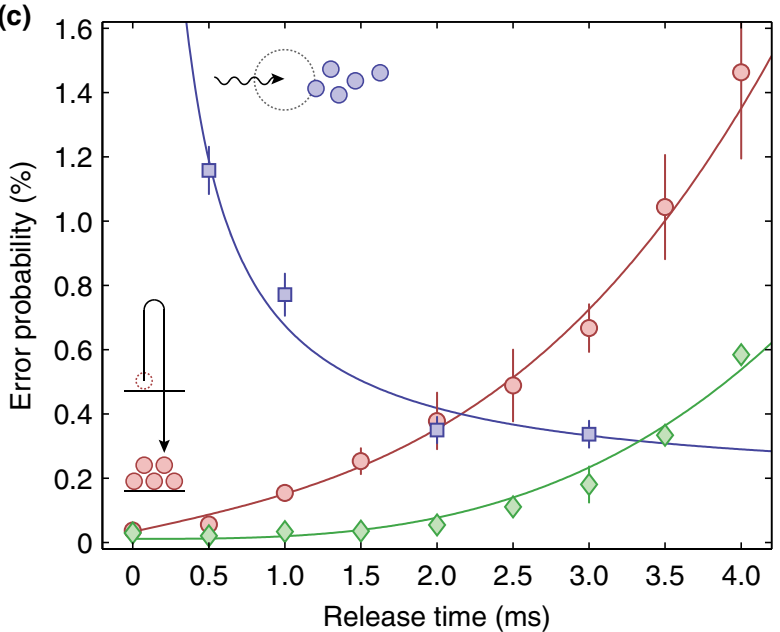

FIG. 4 (color online). Efficiency of state-selective detection via radiation pressure. (a) Level scheme of the ${ }^{87} \mathrm{Rb} D_{2}$ transition. The push beam accelerates atoms in $|F=2\rangle$ out of the capture volume while the MOT beams and repumper are off. The atoms can be prepared in $|F=1\rangle$ via optical pumping. (b) Three example measurements of the $|F=2\rangle$ push efficiency, detecting the atom number before and after the push pulse (dashed line). (c) The measured error rate without push pulse (diamonds) is consistent with isotropic thermal expansion (green line). The errors for $|F=2\rangle$ atoms (squares) can be qualitatively understood based on an analysis of the rate of depumping from $|F=2\rangle$ to $|F=1\rangle$ including the effects of imperfect push beam polarization, and imperfect state preparation (blue line). The error rate for atoms prepared in $|F=1\rangle$ (circles) includes both the effects of thermal expansion and off-resonant optical pumping (red line). All error bars represent 1- $\sigma$ statistical uncertainties.

frame), which imparts momentum to the $|F=2\rangle$ atoms, while ideally leaving the $|F=1\rangle$ atoms unaffected. We measure the overall error probability for the two cases when the atoms are prepared in either $|F=2\rangle$ or $|F=1\rangle$ via optical pumping. The release time with equal error probability for both states is found to be $2.2 \mathrm{~ms}$, where we measure an average fidelity of $99.6(1) \%$, sufficient for detecting the state of 250 atoms with single-atom resolution.

In summary, we have shown single-atom resolution for fluorescence measurements of up to 1200 atoms. Our

results show that a MOT, with high trap depths, low atom density, and high photon scattering rate, is a near ideal trap for precise fluorescence measurements. Since the basic experimental techniques used here are common to many cold-atom experiments, an atom detector with this level of performance could be implemented in many contexts. In addition, our noise analysis is relevant for fluorescence measurements in other atom traps such as optical dipole traps, where we expect both atom loss from light-assisted collisions and photon shot noise to be more severe constraints. In the present work, we demonstrated stateselective detection using radiation pressure to separate two hyperfine states, counting the atoms of one state that remain in the trap volume. However, for many experiments it would be advantageous to simultaneously detect the atom number in two or more subensembles. For this, we envision a system where atoms in the relevant quantum states are separated spatially then individually trapped and detected via fluorescence measurements. We are currently developing this capability in our experiment. When combined, for example, with Stern-Gerlach separation, this will enable measurements of spin-entangled BECs at the Heisenberg limit.

The authors acknowledge experimental contributions by Patrick Reiser. This work was supported by the Heidelberg Center for Quantum Dynamics, Forschergruppe FOR760 of the Deutsche Forschungsgemeinschaft, and the FETOpen project QIBEC (Contract No. 284584). D. B.H. acknowledges support from the Alexander von Humboldt Foundation, and I.S. from the International Max Planck Research School (IMPRS-QD).

*atomcounting@matterwave.de

[1] V. Giovannetti, S. Lloyd, and L. Maccone, Science 306, 1330 (2004).

[2] R. H. Hadfield, Nat. Photonics 3, 696 (2009).

[3] W. Neuhauser, M. Hohenstatt, P.E. Toschek, and H. Dehmelt, Phys. Rev. A 22, 1137 (1980).

[4] J. J. Bollinger, W. M. Itano, D. J. Wineland, and D. J. Heinzen, Phys. Rev. A 54, R4649 (1996).

[5] M. Kitagawa and M. Ueda, Phys. Rev. A 47, 5138 (1993).

[6] J. Ma, X. Wang, C. Sun, and F. Nori, Phys. Rep. 509, 89 (2011).

[7] C. Klempt, O. Topic, G. Gebreyesus, M. Scherer, T. Henninger, P. Hyllus, W. Ertmer, L. Santos, and J. J. Arlt, Phys. Rev. Lett. 104, 195303 (2010).

[8] C. Gross, H. Strobel, E. Nicklas, T. Zibold, N. Bar-Gill, G. Kurizki, and M. Oberthaler, Nature (London) 480, 219 (2011).

[9] C. Hamley, C. Gerving, T. Hoang, E. Bookjans, and M. Chapman, Nat. Phys. 8, 305 (2012).

[10] C. F. Ockeloen, A. F. Tauschinsky, R. J. C. Spreeuw, and S. Whitlock, Phys. Rev. A 82, 061606 (2010).

[11] W. Muessel, H. Strobel, M. Joos, E. Nicklas, I. Stroescu, J. Tomkovi, D. Hume, and M. Oberthaler, Appl. Phys. B 113, 69 (2013). 
[12] R. Bücker, A. Perrin, S. Manz, T. Betz, C. Koller, T. Plisson, J. Rottmann, T. Schumm, and J. Schmiedmayer, New J. Phys. 11, 103039 (2009).

[13] Z. Hu and H. J. Kimble, Opt. Lett. 19, 1888 (1994).

[14] W. Alt, D. Schrader, S. Kuhr, M. Müller, V. Gomer, and D. Meschede, Phys. Rev. A 67, 033403 (2003).

[15] F. Serwane, G. Zürn, T. Lompe, T. Ottenstein, A. Wenz, and S. Jochim, Science 332, 336 (2011).

[16] T. Grünzweig, A. Hilliard, M. McGovern, and M.F. Andersen, Nat. Phys. 6, 951 (2010).

[17] T. Puppe, I. Schuster, A. Grothe, A. Kubanek, K. Murr, P. W. H. Pinkse, and G. Rempe, Phys. Rev. Lett. 99, 013002 (2007).

[18] R. Gehr, J. Volz, G. Dubois, T. Steinmetz, Y. Colombe, B. L. Lev, R. Long, J. Estève, and J. Reichel, Phys. Rev. Lett. 104, 203602 (2010).
[19] J. Goldwin, M. Trupke, J. Kenner, A. Ratnapala, and E. Hinds, Nat. Commun. 2, 418 (2011).

[20] H. Zhang, R. McConnell, S. Ćuk, Q. Lin, M. H. SchleierSmith, I. D. Leroux, and V. Vuletić, Phys. Rev. Lett. 109, 133603 (2012).

[21] K. D. Nelson, X. Li, and D. S. Weiss, Nat. Phys. 3, 556 (2007).

[22] W.S. Bakr, A. Peng, M.E. Tai, R. Ma, J. Simon, J. I. Gillen, S. Foelling, L. Pollet, and M. Greiner, Science 329, 547 (2010).

[23] J. F. Sherson, C. Weitenberg, M. Endres, M. Cheneau, I. Bloch, and S. Kuhr, Nature (London) 467, 68 (2010).

[24] See Supplemental Material at http://link.aps.org/ supplemental/10.1103/PhysRevLett.111.253001 for further details about the experiment, noise model, and fidelity estimates. 